\title{
IMPLANTAÇÃO ESTEREOTÁXICA DE ELETRODOS PROFUNDOS POR RESSONÂNCIA MAGNÉTICA PARA CIRURGIA DE EPILEPSIA
}

\author{
MURILO S. MENESES*, FLÁVIA R. FOLLADOR**, WALTER O. ARRUDA**, \\ HERALDO L. SANTOS**, DÉBORA YONESAWA***, SONIVAL C. HUNHEVICZ*
}

\begin{abstract}
RESUMO - Apresentamos o caso de uma paciente com epilepsia refratária ao tratamento medicamentoso e submetida à monitorização em vídeo-eletrencefalografia por eletrodos de profundidade intracerebrais. A história, o exame clínico, a ressonância magnética (RM), a vídeo-eletrencefalografia e o estudo neuropsicológico não foram suficientes para a determinação da área cerebral de origem das crises convulsivas. Eletrodos de profundidade intracerebrais colocados por estereotaxia guiada por RM possibilitaram o registro de forma muito clara da atividade epileptiforme, determinando com precisão a área cerebral epileptogênica a ser removida por cirurgia. Após lobectomia temporal anterior direita com amígdalo-hipocampectomia realizada há três meses, a paciente permanece sem crises convulsivas. Segundo informações obtidas durante o último Congresso da Liga Brasileira de Epilepsia, esta é a primeira cirurgia estereotáxica para colocação de eletrodos de profundidade intracerebrais em epilepsia no Brasil.
\end{abstract}

PALAVRAS-CHAVE: cirurgia de epilepsia, eletrodos de profundidade, estereotaxia, ressonância magnética.

\section{MRI-guided stereotactic implantation of depth electrodes in epilepsy surgery}

ABSTRACT - We present the case of a 40-year-old woman with refractory epilepsy since aged 18, who was submitted to video-EEG monitoring with intracerebral depth electrodes. The clinical history and examination, magnetic resonance image (MRI), video-EEG and neuropsychological study did not allow the determination of the cerebral onset of epileptic seizures. Depth electrodes inserted by MRI-guided stereotaxis allowed the recording of the epileptic activity and thus showed quite accurately the area of the brain to be surgically resected. She underwent a right anterior temporal lobectomy with amygdalohippocampectomy. The immediate postoperative period was uneventful and she is without epileptic seizures after three months of follow-up. The average preoperative free-seizure period was two weeks. To our knowledge, this is the first stereotactic surgery for insertion of depth intracerebral electrodes in epilepsy in Brazil.

KEY WORDS: epilepsy surgery, depth electrodes, stereotaxis, magnetic resonance imaging.

A condição sine qua non para seleção de pacientes para cirurgia de epilepsia é a demonstração da localização elétrica do início de uma crise convulsiva espontânea em pacientes com epilepsia refratária ao tratamento medicamentoso ${ }^{1}$. Evidentemente, a história e o exame clínico, a avaliação neuropsicológica e exames, como ressonância magnética (RM) e estudo do fluxo sanguíneo cerebral (p. ex. SPECT), são muito importantes na indicação operatória. Havendo concordância das alterações encontradas nesses estudos com os achados no vídeo-eletrencefalograma (v-EEG), não há necessidade de avaliação invasiva. Entretanto, o eletrencefalograma (EEG) de escalpo é menos preciso e pode não ser suficiente para determinar a área epileptogênica. Além disso, os estímulos elétricos levam

Unidade de Cirurgia de Epilepsia, Hospital das Nações de Curitiba: *Neurocirurgião, **Neurologista, ***Neuropsicóloga. Aceite: 2-abril-1999. 
um certo tempo para serem captados no escalpo e, em certos casos, com o início da crise convulsiva, os artefatos musculares se sobrepõem ao traçado eletrencefalográfico, impedindo a determinação do início do evento epiléptico. Nessas situações, métodos invasivos, como eletrodos de profundidade, podem ser indicados.

Relatamos o caso de uma paciente tratada de epilepsia cirurgicamente, após confirmação da área epileptogênica por eletrodos intracerebrais. Segundo informações obtidas durante o último Congresso da Liga Brasileira de Epilepsia ${ }^{2}$, essa técnica não havia sido ainda utilizada no nosso meio, fazendo deste caso o primeiro implante de eletrodos intracerebrais no Brasil para avaliação pré-operatória em cirurgia de epilepsia.

\section{RELATO DO CASO}

CAK, 40 anos, feminina, casada, do lar, natural e procedente de Curitiba, PR. Paciente com história de crises convulsivas com início aos 18 anos de idade. Durante sua primeira gestação apresentou quadro de eclâmpsia, evoluindo posteriormente com coma e crises convulsivas. Houve melhora do quadro clínico logo após a interrupção do parto, mas continuou apresentando crises convulsivas desde então. Essas crises ocorriam com frequência aproximadamente quinzenal, sendo caracterizadas por início com sintoma de mal estar epigástrico e sensação de medo inexplicados. Havia a seguir perda da consciência, ausência de contato com o meio, e apresentava automatismos oromandibulares do tipo mastigatório bem como automatismos manuais do tipo arrumação e limpeza de roupas. Permanecia dessa forma por cerca de 2 minutos e posteriormente voltava a falar adequadamente, com queixas de cefaléia e agitação no período pós-ictal. Eventualmente tinha crises generalizadas tônico-clônicas na evolução das crises parciais anteriormente descritas, fato este que não acontece há alguns anos.

Havia feito uso de fenobarbital na dose máxima de $200 \mathrm{mg} / \mathrm{d}$, fenitoína em dose que não recorda, valproato de sódio também em dose que não recorda, carbamazepina na dose máxima de $1200 \mathrm{mg} / \mathrm{d}$, lamotrigina $200 \mathrm{mg} / \mathrm{d}$ e vigabatrina, isolados e em associações diversas, sem resultado satisfatório. Estava em tratamento com oxcarbazepina $1800 \mathrm{mg} / \mathrm{d}$ associada com clobazam $40 \mathrm{mg} / \mathrm{d}$ e ainda assim mantinha as crises quinzenais.

A paciente negou história anterior de outras doenças, bem como de convulsão febril na infância. A história familiar não apresentava dados dignos de nota.

Os exames físico segmentar e neurológico mostravam-se normais.

Vários exames de EEG interictal foram realizados, demostrando atividade epileptiforme do tipo pontas, de moderada e elevada amplitude projetadas na região temporal direita e esquerda, assíncronas, frequentes, por vezes com predomínio temporal esquerdo. O exame de RM do encéfalo evidenciou diminuição de volume e alteração de sinal nas estruturas hipocampais direitas, sugestivas de esclerose mesial temporal direita (Figs 1 e 2). A avaliação neuropsicológica evidenciou sinais de perda de memória, com déficit principal de memória vísuo-espacial.

O exame de v-EEG prolongado com o registro de vários eventos ictais, todos semelhantes clínicamente entre si, apresentou características semiológicas de crise parcial complexa mas com achado eletrencefalográfico por registro com eletrodos de escalpo mostrando início muito sobreposto por artefatos de movimento, os quais impediram a sua regionalização. A utilização de eletrodos esfenoidais não auxiliou a avaliação. Na evolução, visibiliza-se atividade ictal um pouco mais evidente no hemisfério cerebral direito, porém sem a possibilidade de definir-se adequadamente sua regionalização.

Foi então optado pela video-monitorização com eletrodos intracerebrais de profundidade, com implante bilateral de eletrodos de Spencer de 8 contatos por meio de cirurgia estereotáxica (Figs 3 e 4). Os exames de raio X (Fig 5) e de tomografia computadorizada (Fig 6) mostram os eletrodos intracerebrais implantados.

Após o procedimento cirúrgico, a paciente foi levada para a unidade de v-EEG, onde foi submetida ao estudo neurofisiológico prolongado. O registro de eventos ictais semelhantes aos anteriores revelou como achado eletrencefalográfico a lateralização do seu início de forma extremamente clara na região temporal direita (Fig 7), sendo que o padrão crítico gráfico iniciava-se cerca de 5 segundos antes do início clínico do evento e 25 segundos antes do início do registro da crise através dos eletrodos de escalpo.

Realizou-se o teste do amital sódico de Wada que mostrou a lateralização da área da linguagem no hemisfério cerebral esquerdo e alteração importante da memória vísuo-espacial.

Após a avaliação pré-operatória descrita, indicou-se tratamento cirúrgico por lobectomia temporal anterior direita com amígdalo-hipocampectomia, realizada há 3 meses. A evolução pós-operatória foi simples e sem 


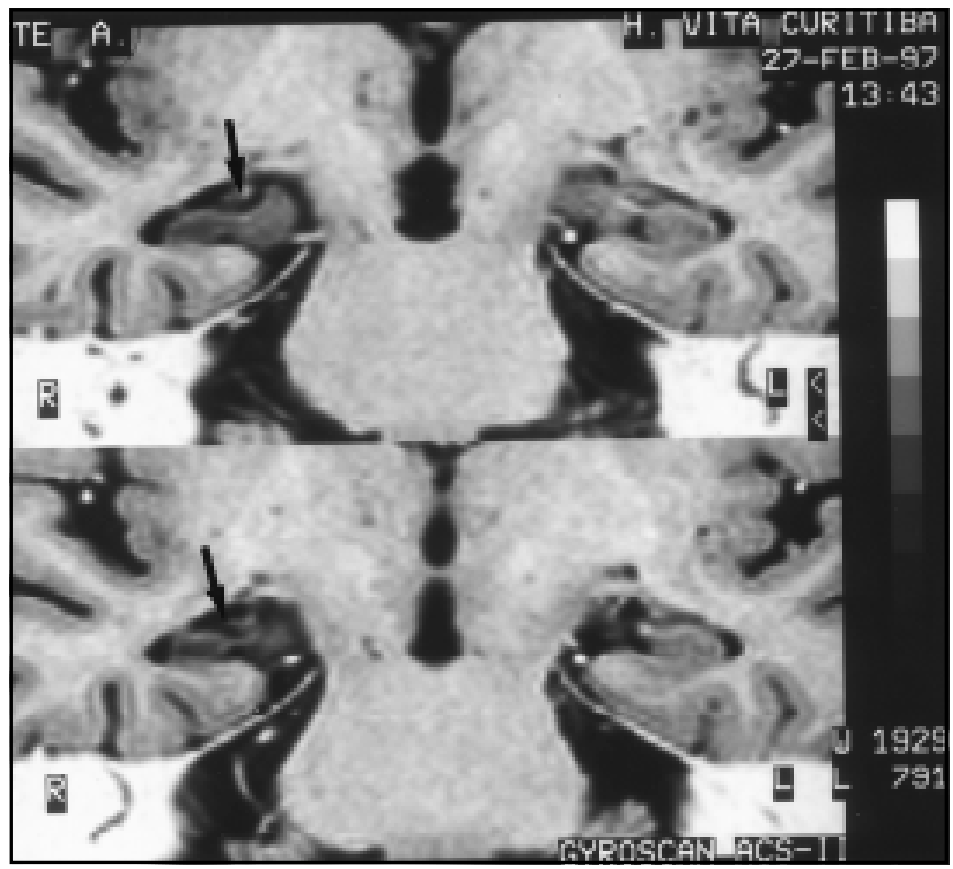

Fig 1. Imagem de ressonância magnética do encéfalo em T2 invertido, no plano coronal, compativel com esclerose mesial temporal direita (flechas).

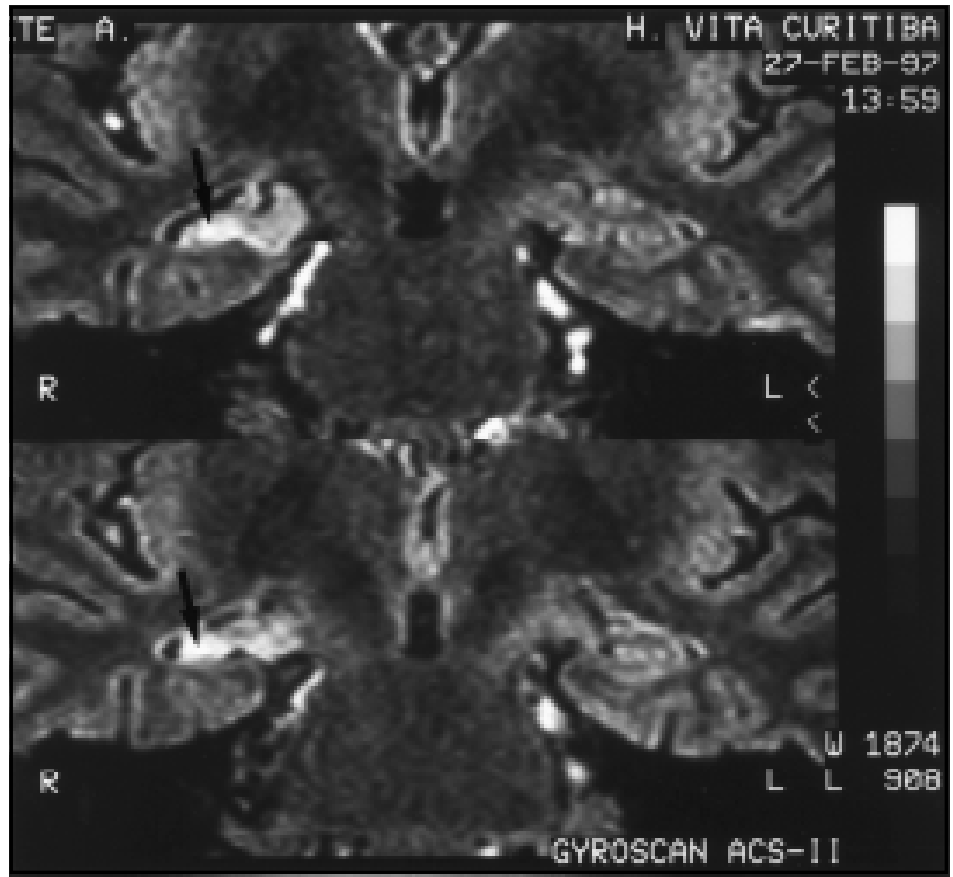

Fig 2. Imagem de ressonância magnética do encéfalo em flair, no plano coronal, compativel com esclerose mesial temporal direita (flechas). 


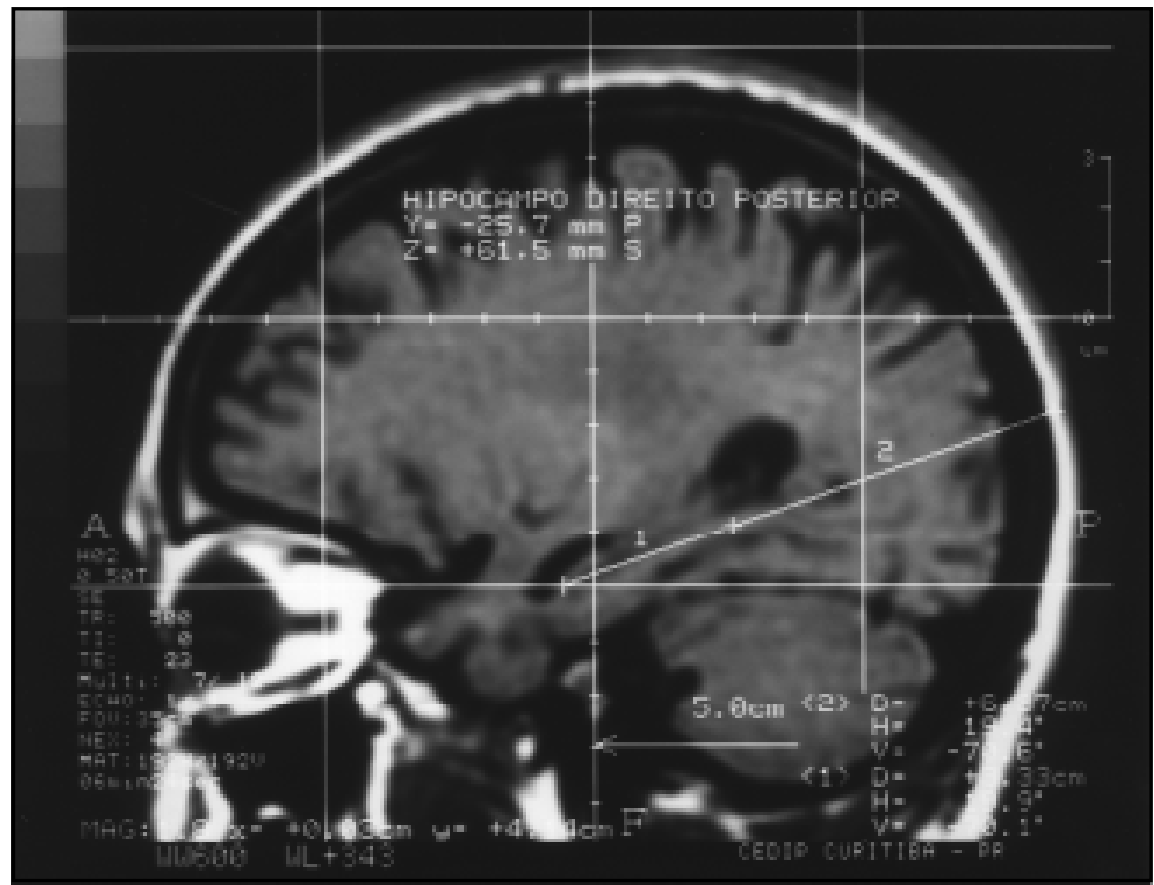

Fig 3. Imagem de ressonância magnética do encéfalo para determinação das coordenadas estereotáxicas para o implante de eletrodo intracerebral no plano sagital.

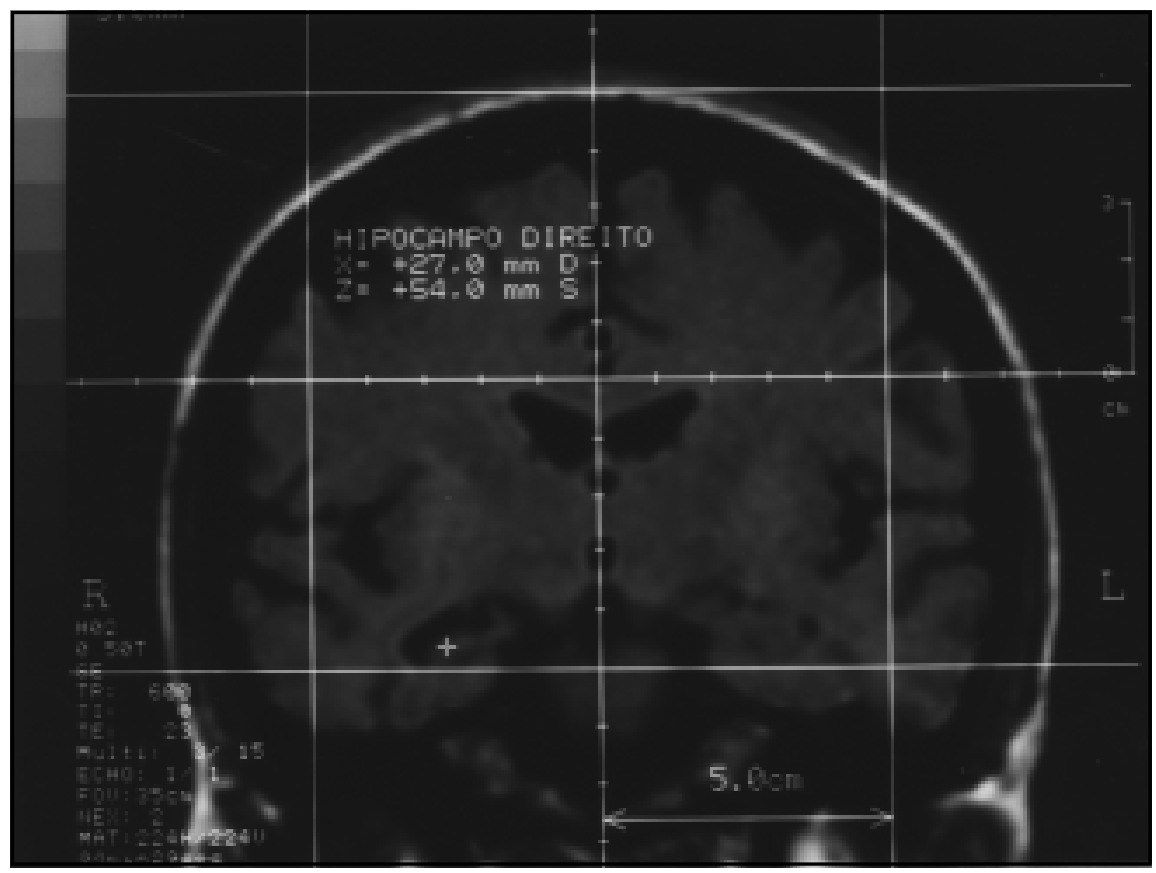

Fig 4. Imagem de ressonância magnética do encéfalo para determinação das coordenadas estereotáxicas do hipocampo direito no plano coronal. 


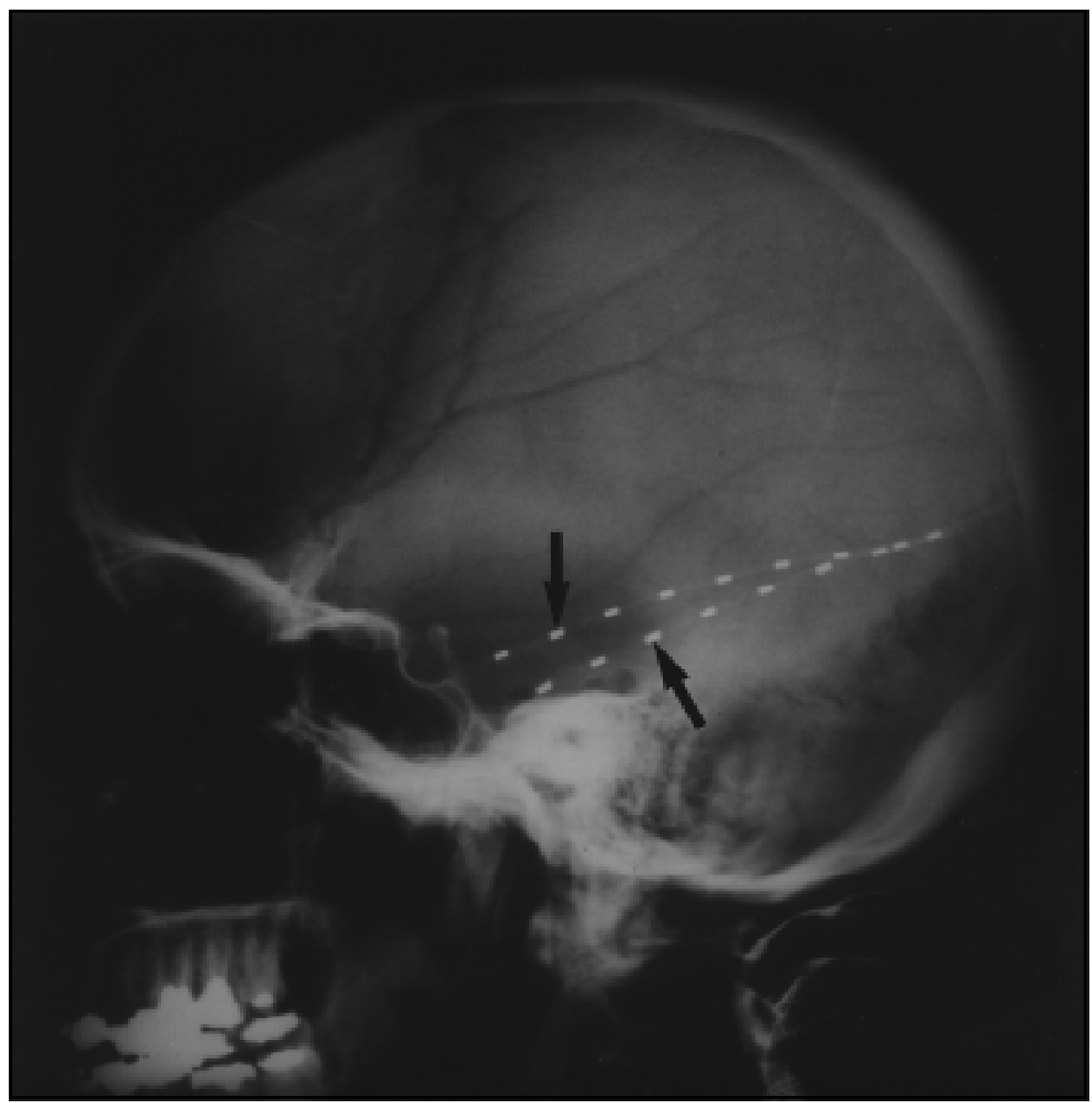

Fig 5. Raio X de crânio em perfil mostrando os eletrodos intracerebrais implantados (flechas).

intercorrências, com desaparecimento total das crises convulsivas desde então. O tratamento medicamentoso atual inclui oxcarbazepina $1800 \mathrm{mg} / \mathrm{d}$ e clobazam $20 \mathrm{mg} / \mathrm{d}$.

\section{Cirurgia de Implante de Eletrodos Intracerebrais}

Segundo a técnica de Spencer e col. ${ }^{3}$, a paciente é submetida a sedação com diprivan e, sob anestesia local (xilocaína 2\%) nos pontos de fixação, o marco estereotáxico é colocado, minutos antes do exame de RM. Este exame mostra as coordenadas estereotáxicas dos dois hipocampos (Fig 2) nos planos coronal, horizontal e sagital, nos seus eixos ântero-posteriores.

No centro cirúrgico e com anestesia local, são realizadas duas incisões occipitais com orifício de trepanação subjacente, com localização pré-determinada por estereotaxia. Após incisão da dura-máter e coagulação do córtex cerebral um eletrodo de Spencer com 8 contatos é introduzido em cada hipocampo, seguindo seu eixo pósteroanterior, conforme as coordenadas estereotáxicas determinadas por RM. Os eletrodos são fixados ao couro cabeludo e conectados aos cabos para monitorização eletrencefalográfica. A paciente é transferida à unidade de v-EEG.

Após os registros eletrencefalográficos de três crises convulsivas, os eletrodos foram removidos sem dificuldades na unidade de v-EEG. 


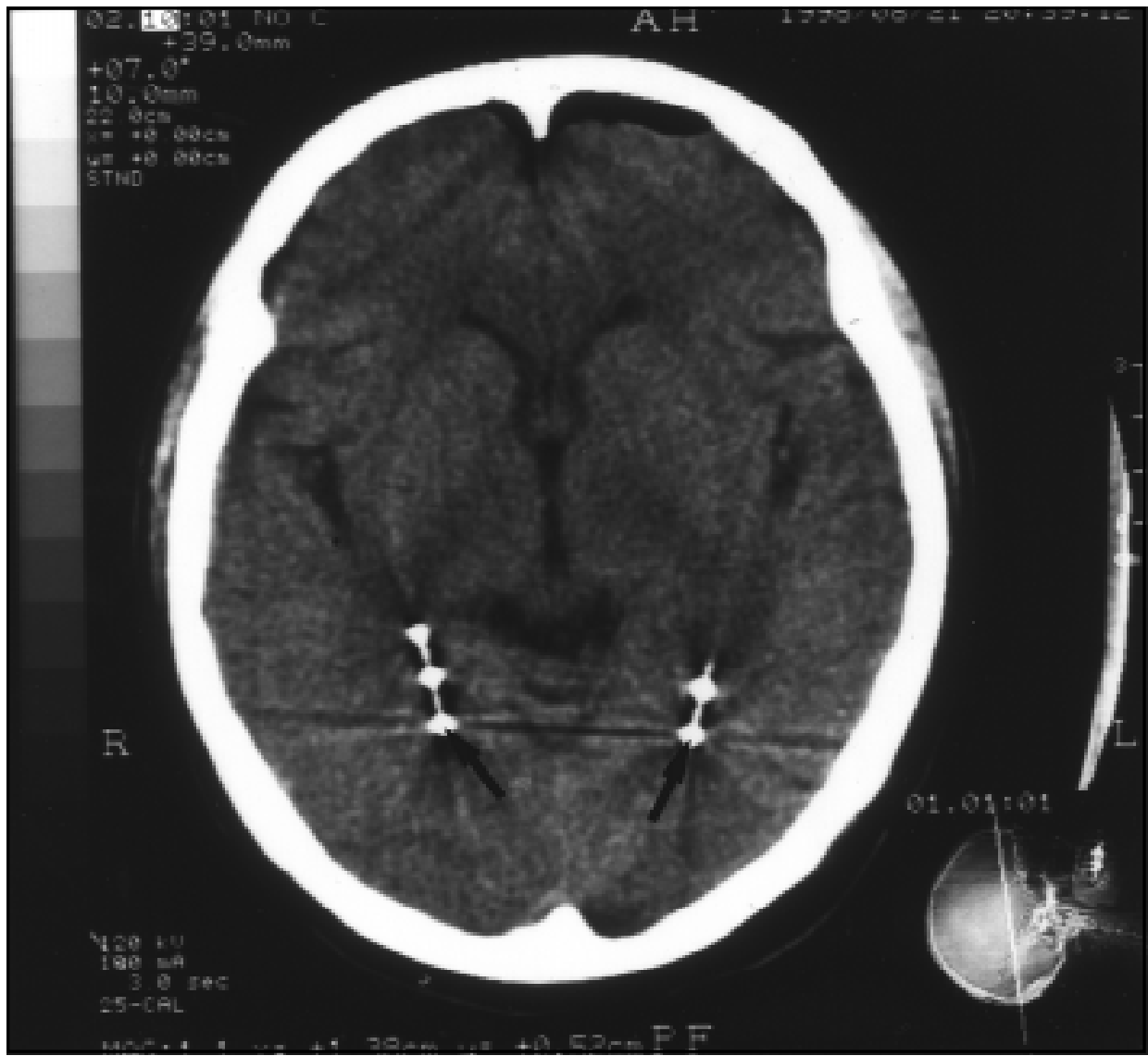

Fig 6. Tomografia computadorizada de crânio mostrando os eletrodos intracerebrais implantados (flechas).

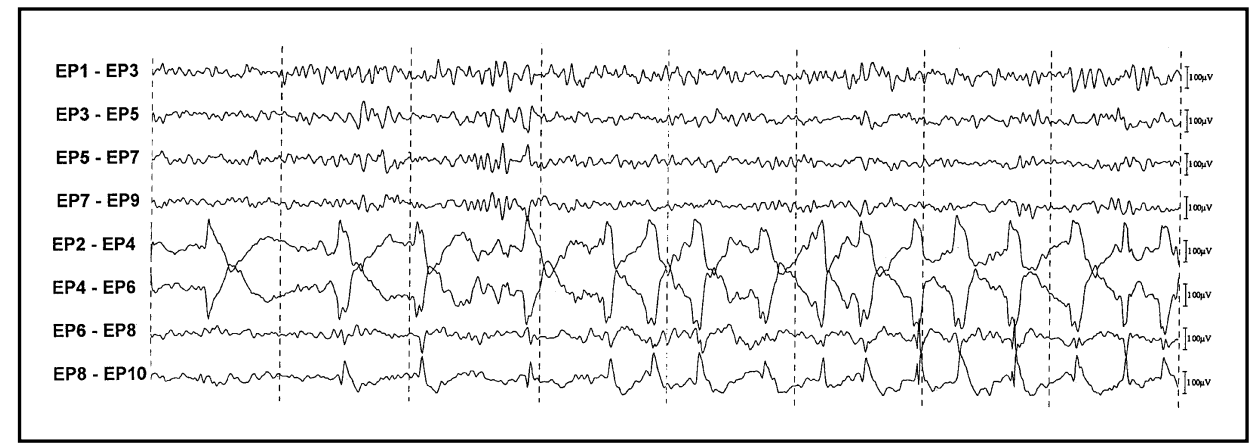

Fig 7. Video-EEG demonstra crise gráfica somente presente nos eletrodos de profundidade a direita (EP2-EP4, EP4-EP6, EP6-EP8, EP8-EP10) em contraposição aos eletrodos profundos à esquerda (EP1-EP3, EP3-EP5, EP5-EP7, EP7-EP9). Durante este periodo os eletrodos de superficie não registraram nenhum tipo de atividade ictal concomitante. A crise clínica ocorre com inicio no mesmo momento que inicia a crise nos eletrodos de profundidade. 


\section{DISCUSSÃO}

Alguns pacientes epilépticos, refratários ao tratamento medicamentoso, apresentam concordância clínica, eletrencefalográfica, neuropsicológica e por exames de imagem e de fluxo sanguíneo cerebral, que permitem uma indicação operatória bastante precisa. Em outros casos, quando não há concordância de dados impõe-se a necessidade de utilizar outros meios que possibilitem melhor definição eletrencefalográfica da área epileptogênica.

Os métodos chamados semi-invasivos, como os eletrodos de forame oval ${ }^{4}$, são opções na tentativa de determinar a área epileptogênica, ao analisar melhor as regiões mesiais dos lobos temporais. Entretanto, frequentemente não são suficientes para a indicação operatória, e representam algum grau de agressão e custo ao paciente. Eletrodos colocados no espaço subdural diretamente sobre o córtex cerebral através de grades ou fitas de eletrodos, permitem uma avaliação pré-operatória detalhada das áreas envolvidas e são excelente opção nos casos de difícil localização, principalmente em regiões extra-temporais. Essas técnicas não devem ser consideradas como concorrentes dos eletrodos intracerebrais e, sim complementares. Na epilepsia temporal, os eletrodos intracerebrais apresentam sensibilidade muito maior na avaliação de estruturas hipocampais que as fitas subdurais colocadas inferiormente ao lobo temporal ${ }^{5}$.

Ross e col. ${ }^{6}$ analisaram retrospectivamente os resultados de eletrodos intracerebrais implantados por estereotaxia guiada por RM e simultânea monitorização com strips subdurais em 50 pacientes. O objetivo desse estudo era de determinar as indicações, eficácia e segurança dessa técnica, utilizada por 3 a 33 dias, com média de 14 dias, nos pacientes em vídeo-EEG. Não houve óbito, infecção ou novo déficit neurológico, e observou-se alta eficácia na determinação da área de origem de crises parciais complexas.

A estereotaxia é técnica que permite atingir uma área intracraniana determinada por um exame de neuroimagem, de forma extremamente precisa, através de pequeno orifício de trepanação realizado geralmente sob anestesia local, com morbidade muito baixa. Esse método é utilizado para biópsia de tumores $^{7,8}$, aspiração de $\operatorname{cistos}^{9,10}$ e hematomas ${ }^{11}$, no tratamento de movimentos anormais ${ }^{12,13}$, psicocirurgia ${ }^{14,15}$ e remoção guiada de lesões profundas e em áreas eloquentes ${ }^{16}$.

Talairach e col. ${ }^{17}$, utilizando seu sistema estereotáxico, descreveram método de implante de eletrodos profundos com uma grade lateral. Dessa forma, um número variável de eletrodos pode ser introduzido perpendicularmente à face externa do cérebro. Originalmente a determinação estereotáxica era realizada com ventriculografia e, devido à existência de vasos principalmente no sulco lateral, com angiografia cerebral. A técnica descrita por Spencer e col. ${ }^{3}$ para epilepsia mesial temporal avalia as áreas hipocampais por um eletrodo implantado por via posterior no eixo dessa estrutura. Como o hipocampo é estrutura alongada no eixo ântero-posterior ${ }^{18}$, este método tem a vantagem de registrar a atividade elétrica em toda sua extensão, auxiliando também na determinação do tamanho da ressecção a ser realizada.

A morbidade das cirurgias estereotáxicas é sabidamente muito baixa, mas a introdução do eletrodo dentro do tecido cerebral deixa visível pela RM o trajeto do eletrodo utilizado, o que poderia potencialmente causar algum déficit neurológico. A avaliação de 17 pacientes, com dois grupos controle, estudados do ponto de vista neuropsicológico antes e após o implante de eletrodos, demonstrou que não houve alteração relacionada ao procedimento ${ }^{5}$.

O uso de ventriculografia e angiografia cerebral para determinação das coordenadas estereotáxicas tem sido substituído por tomografia computadorizada ${ }^{19}$ e $\mathrm{RM}^{20}$, que são menos agressivas, com menores riscos relacionados à injeção de contraste e mais precisas. Optamos pela RM porque este exame permite a realização de imagens em diferentes planos do espaço, apresenta melhor resolução de imagem e permite a visibilização nítida das estruturas mesiais do lobo temporal.

Os eletrodos intracerebrais implantados por cirurgia estereotáxica guiada por RM representam uma excelente opção na avaliação pré-operatória de certos pacientes com epilepsia refratária, possibilitando a determinação precisa da origem elétrica das crises convulsivas de forma segura e orientando claramente a ressecção a ser realizada. 


\section{REFERÊNCIAS}

1. Spencer SS, Katz A. Arriving at the surgical options for intractable seizures. Semin Neurol 1990;10:422-430.

2. Sakamoto AC. O valor do EEG ictal intracrâneo. Neurofisiologia Clínica nas Epilepsias Refratárias. XXIII Reunião da Liga Brasileira de Epilepsia. Congresso Brasileiro de Epilepsia. Porto Alegre, 1998.

3. Spencer SS, So NK, Williamson PD et al. Depth electrodes. In Engel J Jr. Surgical treatment of the epilepsies. New York: Raven Press, 1993;359-376.

4. Wieser HG. Semi-invasive EEG: foramen ovale electrodes. In: Luders HO. Epilepsy surgery, New York: Raven Press, 1992;361-370.

5. Spencer SS, Williamson PD, Spencer DD, Mattson RH. Combined depth and subdural electrode investigation in uncontrolled epilepsy. Neurology 1990;40: 74-79.

6. Ross DA, Brunberg JA, Drury I, Henry TR. Intracerebral depth electrode monitoring in partial epilepsy: the morbidity na efficacy of placebo using magnetic resonance image-guided stereotactic surgery. Neurosurgery 1996;39:327-334.

7. Meneses MS, Mariushi WM, Hunhevicz SC et al. Biópsia estereotáctica para tumores intracranianos. Dendrito 1997;4:30-32.

8. Meneses MS, O’Neill BP, Kelly PJ. Biópsia estereotáxica para linfomas primários do sistema nervoso central. Arq Neuropsiquiatr, 1992;50:319-323.

9. Meneses MS, Arruda WO, Ramina R. Third ventricular cysticercal cyst mimicking a colloid cyst. Neurosurgery 1996;39:623.

10. Meneses MS, Kelly PJ. Microcirurgia estereotáxica para remoção radical de cistos colóides do terceiro ventrículo. Arq Bras Neurocirurg 1992;11:69-75.

11. Meneses MS, Coelho MN, Tsubouchi MH, et al. Tratamento cirúrgico dos hematomas intraparenquimatosos (supra e infratentoriais) espontâneos. In Gagliardi RJ. Doenças cerebrovasculares: condutas. São Paulo: Geo-gráfica, 1996:243-258.

12. Meneses MS, Teive HAG. Doença de Parkinson. Rio de Janeiro: Guanabara Koogan, 1996:142-164.

13. Meneses MS, Teive HAG, Narata AP et al. Talamotomia estereotáxica para hemibalismo. Rev Bras Neurol 1997;34:7-9.

14. Meneses MS, Arruda W.O. Magnetic resonance image-guided stereotactic cingulotomy for intractable psychiatric disease. Neurosurgery 1998;42:432-433.

15. Meneses MS, Arruda WO, Milano JB. Psicocirurgia: passado, presente e futuro. Rev Bras Neurol 1997;33:189-193.

16. Meneses MS, Russ HHA, Coelho MN et al. Cirurgia estereotáctica para processos expansivos intracranianos: diagnóstico e tratamento. Arq Neuropsiquiatr 1996;54:64-70.

17. Talairach J, Bancaud J, Szikla G, et al. Approche nouvelle de la neurochirurgie de l'épilepsie: méthodologie stéréotaxique et résultats thérapeutiques. Neurochirurgie 1974;20:1:240.

18. Arruda WO. Sistema límbico. In Meneses MS. Neuroanatomia aplicada. Rio de Janeiro: Guanabara-Koogan, 1999:273-293.

19. Van Roost D, Solymosi L, Schramm J, et al. Depth electrode implantation in the length axis of the hippocampus for the presurgical evaluation of mesial temporal lobe epilepsy: a computed tomography-based stereotactic insertion technique and its accuracy. Neurosurgery 1998;43:819-827.

20. Meneses MS, Arruda WO, Hunhevicz SC, et al. Comparison of MRI-guided and ventriculography-based stereotactic surgery for Parkinson's disease. Arq Neuropsiquiatr 1998,55:547-552. 